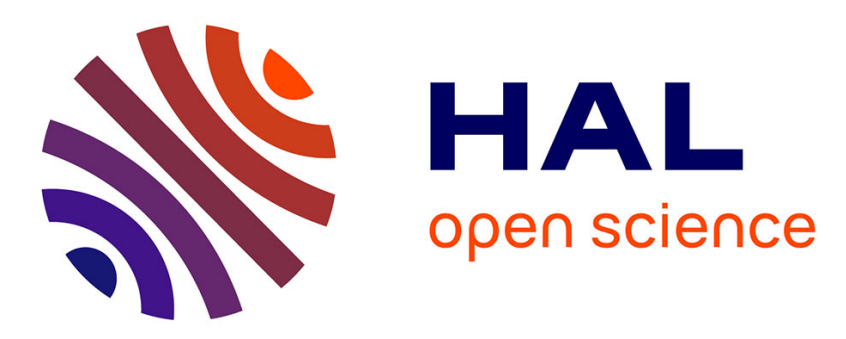

\title{
Fault detection in flight guidance dynamics based on differential flatness
}

Rabah Fellouah, Wen Chi Lu, Felix Mora-Camino, Andrei Doncescu

\section{To cite this version:}

Rabah Fellouah, Wen Chi Lu, Felix Mora-Camino, Andrei Doncescu. Fault detection in flight guidance dynamics based on differential flatness. DASC 2006, 25th IEEE/AIAA Digital Avionics Systerms Conference, Oct 2006, Portsmouth, United States. pp 1-8, 10.1109/DASC.2006.313721 . hal-00938131

\section{HAL Id: hal-00938131 \\ https://hal-enac.archives-ouvertes.fr/hal-00938131}

Submitted on 23 Jun 2014

HAL is a multi-disciplinary open access archive for the deposit and dissemination of scientific research documents, whether they are published or not. The documents may come from teaching and research institutions in France or abroad, or from public or private research centers.
L'archive ouverte pluridisciplinaire HAL, est destinée au dépôt et à la diffusion de documents scientifiques de niveau recherche, publiés ou non, émanant des établissements d'enseignement et de recherche français ou étrangers, des laboratoires publics ou privés. 


\title{
FAULT DETECTION IN FLIGHT GUIDANCE DYNAMICS BASED ON DIFFERENTIAL FLATNESS
}

\author{
Rabbah Fellouah, Wen Chi Lu, Andrei Doncescu, Felix Mora-Camino, ENAC and LAAS du CNRS, \\ Toulouse, France
}

\begin{abstract}
In this paper, flight guidance dynamics are shown to be implicit differentially flat with respect to the inertial position of an aircraft. This proves the existence of a set of relations between these flat outputs and the state variables representative of flight guidance dynamics and between these flat outputs and the basic inputs to flight guidance dynamics. A neural network is introduced to obtain, from the actual trajectory, nominal flight parameters which can be compared with actual values to detect abnormal behaviour.
\end{abstract}

\section{Nomenclature}

$g$ :gravitational acceleration, $m \cdot s^{-2}, N_{1}$ : fan rotational speed of a turbofan engine, RPM (roundper-minute), $\Omega$ : shaft-rotational speed of a pistonpropeller engine, $R P M$ (round-per-minute), $m$ :aircraft mass, $\mathrm{kg}, L, D, T$ :lift, drag, and thrust, $N$, $V_{a}:$ airspeed, $m \cdot s^{-1}, V:$ inertial airspeed, $m \cdot s^{-1}$, $\mathrm{x}, \mathrm{y}, \mathrm{z}$ : aircraft position in the inertial frame, $m$, $\underline{X}, \underline{U}, \underline{Z}$ : state vector, input vector, and flat output vector, $F, G$ : continuous mappings, $: \alpha, \beta$ : angleof-attack, sideslip angle, $\mathrm{rad}, p, q, r$ angular rates in body-axis frame, $\mathrm{rad} \cdot \mathrm{s}^{-1}, \phi, \theta, \psi$ : Euler angles (roll, pitch, and yaw), $\mathrm{rad}, \gamma$ : flight-path angle, $\mathrm{rad}$, $\delta_{a}, \delta_{e}$ : aileron, and elevator settings, $\mathrm{rad}, \delta_{T}$ :throttle setting, $\delta_{T} \in[0,1]$.

\section{Introduction}

The sustained increase of air transportation during the last decades has led to severe air traffic saturation and environmental problems and new needs for aircraft guidance applications have appeared: traffic avoidance and relative guidance maneuvers, formation flight, continuous descent approaches, etc. Differential flatness, a property of some dynamic systems, introduced by Fliess et al. from the theory of differential geometry, has made possible the development of new tools to control and diagnose complex nonlinear systems. Many dynamic non linear systems have been proved to be differentially flat. In the aerospace field, some authors have considered the differential flatness of the dynamics of vertical-take-off-and-landing (VTOL) vehicles, other have considered the differential flatness of relative and formation flight dynamics for different types of flying devices. In particular, some papers have investigated the differential flatness [1] [2] [3] of conventional aircraft dynamics, while few have considered separately the flatness property of the flight guidance and the attitude dynamics of a rigid aircraft.

In this paper, a neural structure is proposed to detect abnormal aircraft behavior. The inertial position of an aircraft is shown to be a differential flat output for its flight guidance dynamics, giving way to their static inversion. Since this differential flatness property is implicit, a neural network is introduced, as a numerical device, to deal with the inversion of the guidance dynamics. Once conveniently structured and trained, the neural network is able to generate in real time estimates of nominal attitude angles and engine regime. These estimates can be compared with actual flight parameters so that abnormal behavior can be detected.

\section{Introduction to Differential Flatness}

Here two definitions of flatness are introduced: One relative to systems for which causal relationships are displayed analytically, it is called explicit flatness and another one, called implicit flatness, where the causal relationships are introduced through implicit functions. The term differential is dropped in the remaining of this paper to avoid cumbersome denominations.

Definition 1: A general nonlinear system given by: 


$$
\underline{\dot{X}}=F(\underline{X}, \underline{U}), \underline{X} \in \mathbf{R}^{n}, \underline{U} \in \mathbf{R}^{m}
$$

where $F$ is a smooth mapping, is said explicitly flat with respect to the output vector $\underline{Z}$, if $\underline{Z}$ is an $m^{\text {th }}$ order vector which can be expressed analytically as a function of the current state, the current input and its derivatives and also such as the state and the input vectors can be expressed analytically as a function of $\underline{Z}$ and its derivatives. Then there exists smooth mappings $G_{X}, G_{U}$, and $G_{Z}$ such as:

$$
\begin{aligned}
& \underline{Z}=G_{Z}\left(\underline{X}, \underline{U}, \ldots, \underline{U}^{(p)}\right) \\
& \underline{X}=G_{X}\left(\underline{Z}, \underline{\dot{Z}}, \ldots, \underline{Z}^{(q)}\right) \\
& \underline{U}=G_{U}\left(\underline{Z}, \underline{Z}, \ldots, \underline{Z}^{(q+1)}\right)
\end{aligned}
$$

which are respectively the output, the state and the input flatness relations, where $p$ and $q$ are integer numbers. Vector $\underline{Z}$ is called a flat output for the nonlinear system Eq.(1). Although there is no systematical way to determine the flat output and its uniqueness as well, the flat output usually possesses some physical meanings. The explicit flatness property is of particular interest for the solution of a control problem when a physically meaningful flat output can be related with its objectives. In many situations, the control problem can be formulated as a flat output trajectory following problem and relation (2.c) must be inverted to get the corresponding nominal control inputs. With respect to fault detection, relation (2.b) as well as relation (2.c) can be used in an analytical redundancy scheme. However, for many systems, no complete analytical models are available to describe their full dynamics. Some of their components make use of input-output numerical devices derived both from theory and from experimental data. In these cases, available theory provides in general the main mathematical properties of these implicit functions while experimental data is used to build accurate input output numerical devices. This happens for instance when flight dynamics modeling is considered either for control or simulation purposes, since in practice the aerodynamic coefficients are obtained through interpolation across sets of data tables.
Definition 2: A nonlinear system given by a general implicit $\mathrm{n}^{\text {th }}$ order state representation:

$$
F(\underline{X}, \underline{X}, \underline{U})=0, \underline{X} \in R^{n}, \underline{U} \in R^{m}
$$

where $F$ is a regular implicit mapping with respect to $\underline{\dot{X}}$, is said implicitly flat over an interior nonempty domain $\Delta \subseteq R^{n+m}$ if it is possible to find an $m^{\text {th }}$ order vector $\underline{Z}$ which meets conditions Eq.(2) and condition:

$$
G\left(\underline{X}, \underline{U}, \underline{Z}, \underline{\dot{Z}}, \ldots, \underline{Z}^{(r)}\right)=\underline{0}
$$

where $G$ is locally invertible over $\Delta$ with respect to $\underline{X}$ and $\underline{U}$ where $r$ is an integer.

Again, vector $\underline{Z}$ is said to be a flat output. The invertibility of $G$ is guaranteed if the determinant of the Jacobian of $G$ is not zero according to the theorem of implicit functions. Eq.(4) and Eq.(5) imply that given a trajectory of the flat output $\underline{Z}$, it is possible to map it numerically into the input space to derive corresponding control command so that one of the more helpful properties of explicitly flat systems is still maintained. Furthermore, any established property for implicitly flat systems will also hold in numerical ground for explicitly flat systems

\section{Flight Guidance Dynamics}

In this study, only the guidance dynamics of transportation aircraft are concerned. It is assumed that the aircraft is equipped with a basic autopilot which deals efficiently with its fast dynamics and controls its attitude $(\theta, \phi, \beta)$ as well as its thrust regime ( $N_{1}$ or $\Omega$ ). It is also supposed that their yaw damper can achieve perfect coordinated-turn motion, so that $\beta$ should remain very small. Here the flight variables $\theta, \phi$ and $N_{1}$ are taken as the inputs for the guidance dynamics as well as the outputs for the fast dynamics around the aircraft body frame when controlled by a basic auto pilot. Figure 1 displays the resulting structure for the whole flight dynamics: 
Thus, in steady wind conditions, the flight guidance dynamics can be expressed in the aerodynamics reference frame as following [4] [5]: $\dot{x}=V_{a} \cos \psi \cos \gamma+w_{x}$, $\dot{y}=V_{a} \sin \psi \cos \gamma+w_{y}$,

$\dot{z}=-V_{a} \sin \gamma+w_{z}$

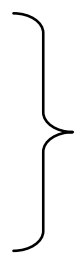

with

$V_{a}=\sqrt{\left(\dot{x}-w_{x}\right)^{2}+\left(\dot{y}-w_{y}\right)^{2}+\left(\dot{z}-w_{z}\right)^{2}}$

and

$\dot{V}_{a}=\frac{-D+T \cos \alpha-m g(-\cos \alpha \sin \theta+\sin \alpha \cos \phi \cos \theta)}{m}$

$\dot{\gamma}=\frac{L \cos \phi+T \sin \alpha-m g(\sin \alpha \sin \theta+\cos \alpha \cos \phi \cos \theta)}{m V_{a}}$

In coordinated-turn motion, the heading rate is related to the bank angle $\phi$ through the following relation:

$$
\dot{\psi}=\left(\frac{g}{V}\right) \tan \phi
$$

The drag $D$ and lift $L$ are respectively considered to be functions of altitude $z$, airspeed $V_{a}$ and angle-of-attack $\alpha$. While the thrust $T$ can be considered to be function of altitude $z$, airspeed $V_{a}$ and engine regime ( $N_{1}$ for a jet and or $\Omega$ for a propeller).

$D=D\left(z, V_{a}, \alpha\right), L=L\left(z, V_{a}, \alpha\right), T=T\left(z, V_{a}, N_{1}\right)$

For local guidance purposes, the flight-path angle $\gamma$ is usually taken as the control parameter.

Meanwhile, through the coordinate transformation, the angle-of-attack $\alpha$ can be expressed as a complex function of $\theta, \phi$, and $\gamma$.Once the bank angle is considered to be small enough, this relation can be reduced to:

$\alpha=\theta-\gamma$

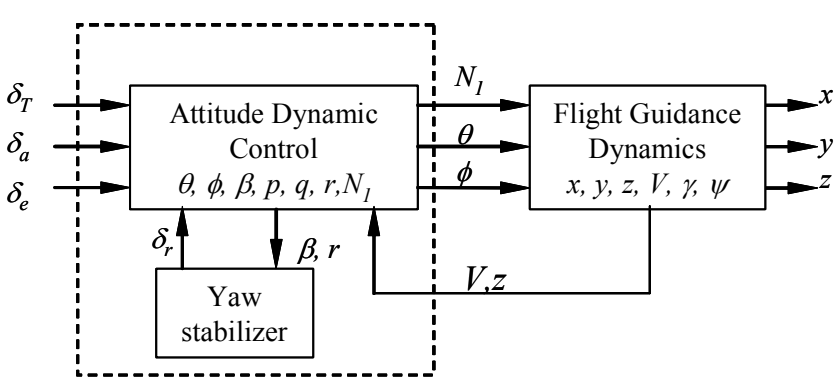

Figure 1. Aircraft Flight Dynamics Structure

\section{Flight Guidance Dynamics and Differential Flatness}

Here it is shown how, starting from the knowledge of $x(t), y(t)$ and $z(t)$, it is possible to express successively all the guidance variables as a function of them and their derivatives [6] [7]. By rearranging the kinematical equations Eq.(6-a), Eq.(7-a), and Eq.(7-b)), and are expressed as:

$$
\gamma=-\sin ^{-1}(\dot{z} / V), \psi=\tan ^{-1}(\dot{y} / \dot{x})
$$

The state variables $V_{a}, \gamma$ and $\psi$, obviously can be functions of the inertial position of aircraft while the control variables satisfy relations (7-a, 7-b, 7-c), where $\alpha$ can be replaced by a function of $\phi, \theta$, and $\gamma$ given in Eq. (9).

$$
\begin{gathered}
(x, y, z) \stackrel{\frac{d}{d t}}{\longrightarrow}\left(V_{e}, V_{a}, \chi, \gamma\right) \\
\left.\downarrow_{\phi}\right|_{\left(\theta, N_{1}\right)} ^{\left(w_{x}{ }^{E}, w_{y}{ }^{E}, w_{z}{ }^{E}\right)}
\end{gathered}
$$

Figure 2. Flatness Propagation through Guidance Dynamics

The following notations are adopted for the position of the center of gravity of the aircraft and for the guidance inputs:

$\underline{Z}=(x, y, z)^{T}$ and $\underline{U}=\left(\theta, \phi, N_{1}\right)$ 
Once the variables in Eq. (7-a), Eq.(7-b) and Eq.(7-c) are replaced by their expressions in $\underline{Z}$ and its first two derivatives, these equations can be rewritten as:

$$
\left.\begin{array}{l}
G_{N_{1}}(\underline{Z}, \underline{\dot{Z}}, \underline{\ddot{Z}}, \underline{U})=0, \\
G_{\theta}(\underline{Z}, \underline{\dot{Z}}, \underline{\ddot{Z}}, \underline{U})=0, \\
G_{\phi}(\underline{Z}, \underline{\dot{Z}}, \underline{\ddot{Z}}, \underline{U})=0
\end{array}\right\}
$$

These implicit functions are locally invertible with respect to the input vector since for normal flight conditions the determinant of their Jacobian is not zero. Then, $\underline{Z}=(x, y, z)^{T}$ is a flat output vector for the considered flight guidance dynamics. The time evolution of these flat outputs represents the trajectory followed by the center of gravity of the aircraft. Then according to the above theory, from the knowledge of this trajectory, it should be possible to find the corresponding inputs.

As a consequence of the above flatness property, given a current trajectory for the flat outputs:

$$
\underline{Z}_{c}(\tau)=\left(x_{c}(\tau), y_{c}(\tau), z_{c}(\tau)\right)^{T}, \tau \in\left[t_{0}, t\right]
$$

the corresponding nominal input values at the instant $t, U_{c}(t)=\left(\phi_{c}(t), \theta_{c}(t), N_{1 c}(t)\right)^{T}$, are solutions of the set of equations:

$$
\left.\begin{array}{l}
G_{N_{1}}\left(\underline{Z}_{c}(t), \underline{\dot{Z}}_{c}(t), \underline{\underline{Z}}_{c}(t), \underline{U}_{c}(t)\right)=0, \\
G_{\theta}\left(\underline{Z}_{c}(t), \underline{\underline{Z}}_{c}(t), \underline{\underline{Z}}_{c}(t), \underline{U}_{c}(t)\right)=0, \\
G_{\phi}\left(\underline{Z}_{c}(t), \underline{\underline{Z}}_{c}(t), \underline{\underline{Z}}_{c}(t), \underline{U}_{c}(t)\right)=0
\end{array}\right\}
$$

Since it seems quite difficult to get an on-line numerical solution to this set of implicit equations, it appears of interest to build a neural network with this objective [8]. Once correctly trained, the neural network should be an input-output device where the inputs are provided by the trajectory followed by the aircraft, while the outputs of the neural network are the corresponding nominal flight parameters. Multilayer neural networks have been shown to be able to perform function approximation with the adequate selection of a neural network structure associated to a learning process. The selection of a structure and of a learning algorithm strongly depend on empirical rules while numerous neural networks structures and learning algorithms are already available. In order to achieve an acceptable accuracy and a sufficient generalization capability, a large amount of training data is necessary.

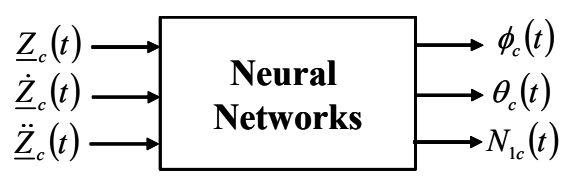

\section{Figure 3. Neural Reference Input Generator for Autopilot}

Here the training data is composed of sets of trajectories for $\underline{Z}$ and $\underline{U}$ which can be provided from either flight test data or even from commercial flight data in which maneuvers are performed manually or by the autopilot engaged basic attitudeholding mode (no guidance loop must be active at that time). Since for modern aircraft onboard navigation systems (ADIRS) are able to estimate with good accuracy the current aircraft position, inertial speed and wind speed, their records can be used as a basis for the training of the neural network.

\section{Strong analytical redundancy relationships}

\section{The case of the state flatness relations}

The state flatness relations of interest here are relations (6-a) which allow, since the acceleration components, the airspeed, the angles $\alpha, \theta, \phi$ and $\psi$ can be measured, to estimate the components of the wind speed which are characteristic of the external perturbation applied to the aircraft. Here different solution approaches with increasing levels of complexity can be developed:

- The above equations can be solved with respect to $\mathrm{w}_{\mathrm{x}}, \mathrm{w}_{\mathrm{y}}$ and $\mathrm{w}_{\mathrm{z}}$, but in this case the measurement errors are not taken into account. Even if using redundancy schemes they can be turned relatively small. We get for instance the estimate: 


$$
\widetilde{w}_{x}=\dot{x}_{m}-V_{a m} \cos \psi_{m} \cos \gamma_{m}
$$

- A raw Kalman filter can be introduced when considering that equations (6.a) are observation equations where the resulting errors are Gaussian white noise. In this case an estimate of wing speed component $i, i \in\{x, y, z\}$, is given by:

$$
\dot{\hat{w}}_{i}=K_{i}\left(\widetilde{w}_{i}-\hat{w}_{i}\right) \quad i \in\{x, y, z\}
$$

where the $K_{i}$ are appropriate Kalman filter gains.

- A more elaborated Kalman filter when linear models of the wind process ( Dryden model for instance) and of the measurement errors are available.

- An adaptive approach when some parameters of the wind process model and of the measurement errors must be estimated on line.

\section{The Case of the Guidance Input Flatness Relations}

Here the neural inversion of relations (7-a, 7-b, 7-c) provides nominal values for $\theta, \phi$ and $\mathrm{N}_{1}: \widetilde{\theta}$, $\widetilde{\phi}$ and $\tilde{N}_{1}$. Considering that the autopilot is designed such that:

$$
\left.\begin{array}{c}
\tau_{\theta} \dot{\theta}+\theta=\theta_{c} \\
\tau_{\phi} \dot{\phi}+\phi=\phi_{c} \\
\tau_{N_{1}} \dot{N}_{1}+N_{1}=N_{1 c}
\end{array}\right\}
$$

it is then possible to propose a set of eight tests to detect any discrepancy with respect to nominal flight guidance dynamics:

$$
\begin{aligned}
& \text { if }\left|\theta_{c}-\widetilde{\theta}-\tau_{\theta} \dot{\theta}\right| \leq \sigma_{\theta} \text { and }\left|\phi_{c}-\widetilde{\phi}-\tau_{\phi} \dot{\phi}\right| \leq \sigma_{\phi} \\
& \text { and }\left|N_{1 c}-\widetilde{N}_{1 c}-\tau_{N_{1}} \dot{N}_{1}\right| \leq \sigma_{N_{1}}
\end{aligned}
$$

then Flight Dynamics are declared nominal

$$
\begin{aligned}
& \text { if }\left|\theta_{c}-\widetilde{\theta}-\tau_{\theta} \dot{\theta}\right| \geq \sigma_{\theta} \text { and }\left|\phi_{c}-\widetilde{\phi}-\tau_{\phi} \dot{\phi}\right| \leq \sigma_{\phi} \\
& \text { and }\left|N_{1_{c}}-\widetilde{N}_{1 c}-\tau_{N_{1}} \dot{N}_{1}\right| \leq \sigma_{N_{1}}
\end{aligned}
$$

then Pitch Dynamics are declared damaged

$$
\text { if }\left|\theta_{c}-\widetilde{\theta}-\tau_{\theta} \dot{\theta}\right| \leq \sigma_{\theta} \text { and }\left|\phi_{c}-\widetilde{\phi}-\tau_{\phi} \dot{\phi}\right| \geq \sigma_{\phi}
$$$$
\text { and }\left|N_{1 c}-\widetilde{N}_{1 c}-\tau_{N_{1}} \dot{N}_{1}\right| \leq \sigma_{N_{1}}
$$

then Bank Dynamics are declared damaged

$$
\begin{aligned}
& \text { if }\left|\theta_{c}-\widetilde{\theta}-\tau_{\theta} \dot{\theta}\right| \leq \sigma_{\theta} \text { and }\left|\phi_{c}-\widetilde{\phi}-\tau_{\phi} \dot{\phi}\right| \leq \sigma_{\phi} \\
& \text { and }\left|N_{1 c}-\widetilde{N}_{1 c}-\tau_{N_{1}} \dot{N}_{1}\right| \geq \sigma_{N_{1}}
\end{aligned}
$$

then Thrust channel is declared damaged

$$
\begin{aligned}
& \text { if }\left|\theta_{c}-\widetilde{\theta}-\tau_{\theta} \dot{\theta}\right| \geq \sigma_{\theta} \text { and }\left|\phi_{c}-\widetilde{\phi}-\tau_{\phi} \dot{\phi}\right| \geq \sigma_{\phi} \\
& \text { and }\left|N_{1 c}-\widetilde{N}_{1 c}-\tau_{N_{1}} \dot{N}_{1}\right| \leq \sigma_{N_{1}}
\end{aligned}
$$

then Pitch and Bank Dynamics are declared damaged

$$
\begin{aligned}
& \text { if }\left|\theta_{c}-\widetilde{\theta}-\tau_{\theta} \dot{\theta}\right| \geq \sigma_{\theta} \text { and }\left|\phi_{c}-\widetilde{\phi}-\tau_{\phi} \dot{\phi}\right| \leq \sigma_{\phi} \\
& \text { and }\left|N_{1 c}-\widetilde{N}_{1 c}-\tau_{N_{1}} \dot{N}_{1}\right| \geq \sigma_{N_{1}}
\end{aligned}
$$

then Longitudinal Dynamics are declared damaged

$$
\begin{aligned}
& \text { if }\left|\theta_{c}-\widetilde{\theta}-\tau_{\theta} \dot{\theta}\right| \leq \sigma_{\theta} \text { and }\left|\phi_{c}-\widetilde{\phi}-\tau_{\phi} \dot{\phi}\right| \geq \sigma_{\phi} \\
& \text { and }\left|N_{1 c}-\widetilde{N}_{1 c}-\tau_{N_{1}} \dot{N}_{1}\right| \geq \sigma_{N_{1}}
\end{aligned}
$$

then Bank and Thrust dynamics are declared damaged

$$
\begin{aligned}
& \text { if }\left|\theta_{c}-\widetilde{\theta}-\tau_{\theta} \dot{\theta}\right| \geq \sigma_{\theta} \text { and }\left|\phi_{c}-\widetilde{\phi}-\tau_{\phi} \dot{\phi}\right| \geq \sigma_{\phi} \\
& \text { and }\left|N_{1_{c}}-\widetilde{N}_{1 c}-\tau_{N_{1}} \dot{N}_{1}\right| \geq \sigma_{N_{1}}
\end{aligned}
$$

then Full Flight Dynamics are declared damaged.

\section{Simulation and Results}

A simulation model of a light aircraft, the Navion, (Schmidt, 1998) [9] with a piston-propeller engine and a simple PID controller for attitude holding has been used for the generation of training data and for validation purposes (see Fig.4).

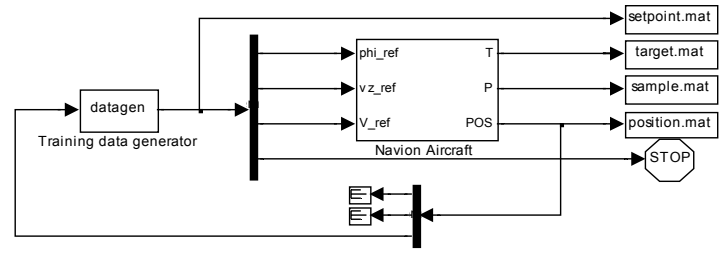

Figure 4. Simulink Generation of Trajectory Training Data for the Navion 
Preliminary simulation results have been obtained in the case of maneuvers in the vertical plane. In this study, the conventional Error-BackPropagation neural network [10] with only one hidden layer has been selected to perform the inversion of flight guidance dynamics, although other neural network structures [11] have been investigated.

The structure of the retained neural network comprises seven inputs nodes, about 90 neurons in the hidden layer with a hyperbolic tangent activation function, and three output nodes with linear transfer functions. The seven inputs are altitude, three components of inertial velocity and three components of inertial acceleration. The three outputs are the pitch $\theta$, the roll $\phi$ and $\Omega$ the engine input signals. Figures 5, 6 and 7 display the training data used over a sample of 3000 data points as well as the corresponding training errors. Figure 8 presents different training experiences while figures 9, 10 and 11 display validation data. It can be observed that learning and validation reach a satisfactory level of accuracy.
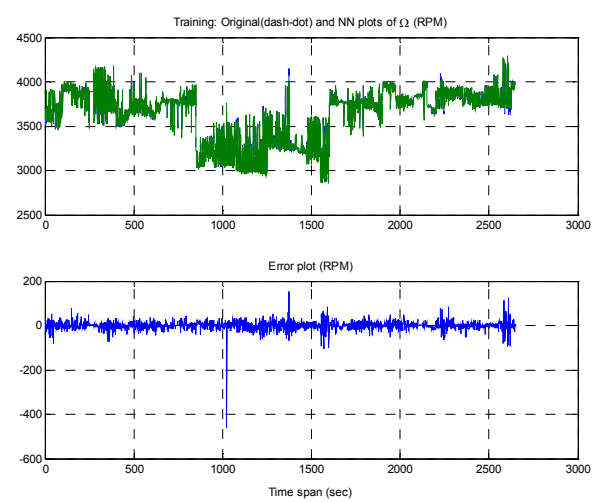

Figure 5. Training Data for the Engine Regime $\Omega$
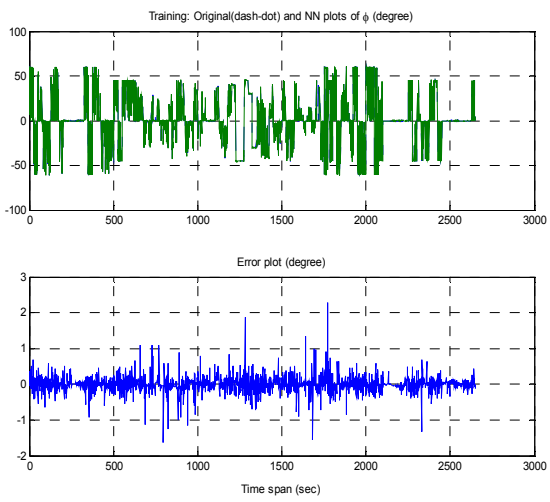

Figure 6. Training Data for the Bank Angle $\phi$
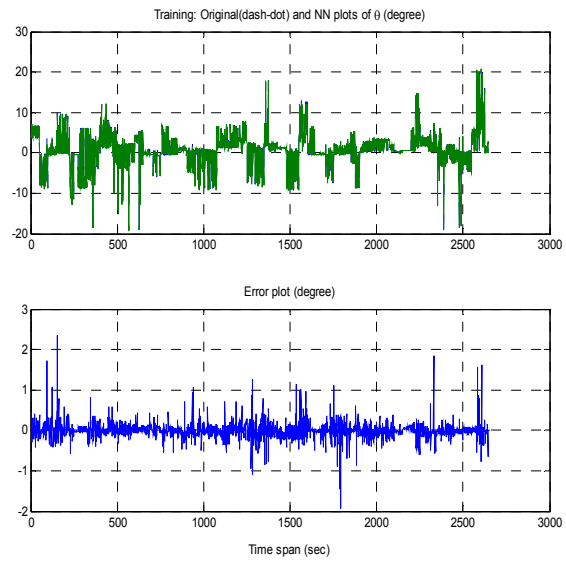

Figure7. Training Data for the Pitch Angle $\theta$

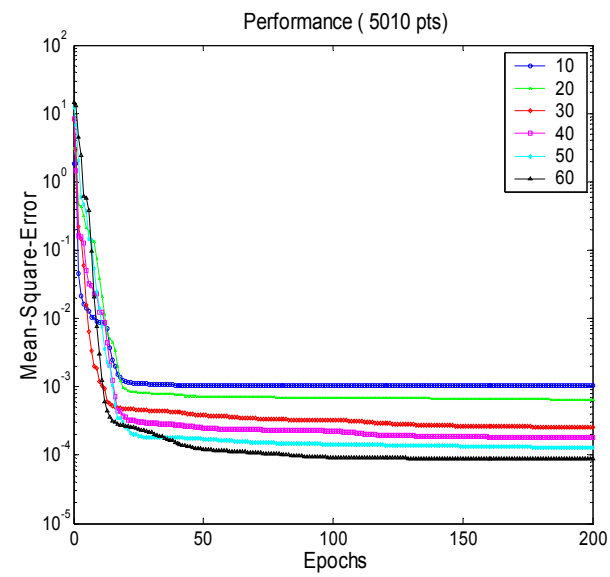

Figure 8. Training Error History for Different Numbers of Neurones in the Hidden Layer 

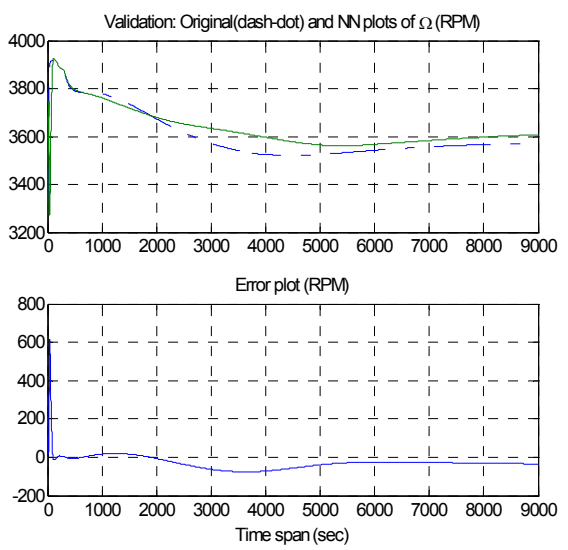

Figure 9. Validation Data for the Engine Regime $\Omega$
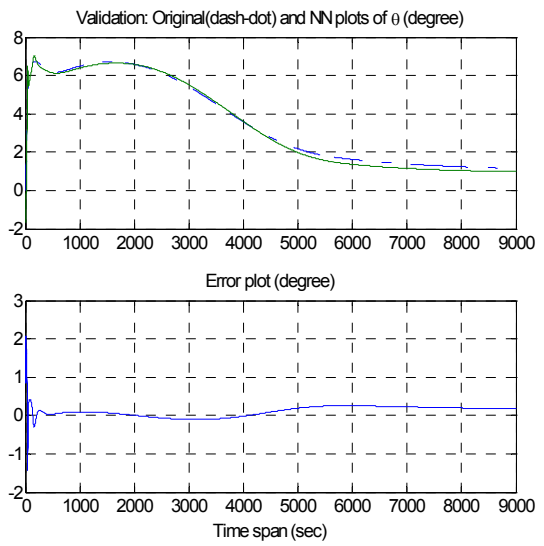

Figure 10. Validation Data for the Bank Angle $\phi$
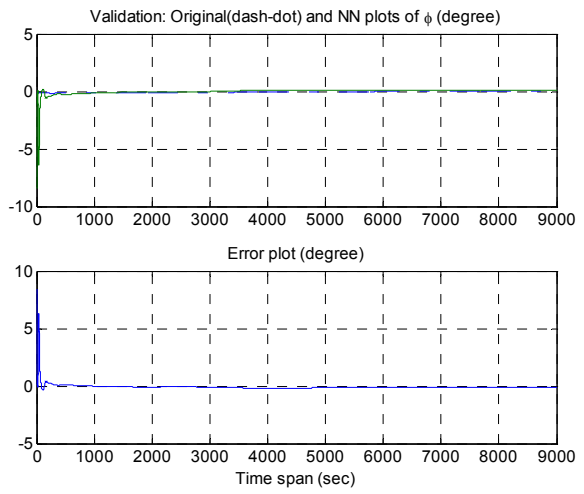

Figure 11. Validation Data of the Pitch Angle $\theta$

\section{Conclusion}

In this communication, differential flatness theory has been considered to provide new implicit relations between trajectory parameters and flight dynamics variables. Neural networks are introduced to capture these implicit relations. The resulting approach appears to provide a possible basis for the development of fault detection systems which should trigger fault tolerant and adaptive schemes to guarantee flight safety as well as trajectory tracking accuracy under damaged flight dynamics conditions.

\section{References}

[1] Fliess, M., J. Lévine, P. Martin, and P. Rouchon, "Flatness and defect of non-linear systems: theory and examples," Int. J. Control, Vol. 61, No. 6, 1995, pp. 1327-1361.

[2] Lavigne, L., F. Cazaurang, B. Bergeon, "Modelling of Longitudinal Disturbed Aircraft Model by Flatness Approach," AIAA Guidance, Navigation, and Control Conference and Exhibit, Texas Austin, USA, 2003.

[3] Martin, P., "Contribution a l'Étude des Systèmes Différentielles plats," Ph.D. Dissertation, L’École des Mines de Paris, France, 1992.

[4] Brian L. Steven, Frank L. Lewis, 1992, Aircraft Control and Simulation, John Wiley \& Sons, Inc., 1992.

[5] Lu, W., F. Mora-Camino, M. de Coligny, K. Achaibou, "Flight Mechanics and Differential Flatness," DINCON 2004, Ilha Solteira, Brasil, pp. 830-839, 2004.

[6] Lu, W., F. Mora-Camino, "Systèmes Plats et La Dynamique du Vol," EDSYS Congres Doctorant, Toulouse, France, 2004.

[7] Neural Network Toolbox for Use with MATLAB ${ }^{\circledR}, 2000$, The MathWorks, Inc.

[8] Haykin, S., Neural Networks, a Comprehensive Foundation, Macmillan Publishing Company, NJ, 1994.

[9] AEROSIM BLOCKSET User's Guide, Unmanned Dynamics, LLC.

[10] Schmidt, Louis V., Introduction to Aircraft Flight Dynamics, AIAA Education Series, 1998. 
[11] Rodríguez, Carmen Elena Quiroga,

"Representation Neuronale de la Dynamique de Vol d'un Aeronef," DEA Technical Report of LAAS du CNRS, 2005. 